\title{
Ultrasound-Guided Multilevel Thoracic
}

\section{Paravertebral Block and Its Efficacy for Surgical Anesthesia During Primary Breast Cancer Surgery}

This article was published in the following Dove Press journal:

Journal of Pain Research

\section{Pawinee Pangthipampai (D) \\ Manoj K Karmakar (ID \\ Banchobporn Songthamwat \\ Jatuporn Pakpirom \\ Winnie Samy}

Department of Anesthesia and Intensive Care, The Chinese University of Hong Kong, Prince of Wales Hospital, Shatin, New Territories, Hong Kong Special Administrative Region of the People's Republic of China
Correspondence: Manoj K Karmakar Department of Anesthesia and Intensive Care, The Chinese University of Hong Kong, Prince of Wales Hospital, Room 042 ID, 4/F, Main Clinical Block and Trauma Centre, Shatin, New Territories, Hong Kong, Special Administrative Region of the People's Republic of China

Tel +852 3505-2735

Fax +852 3505-2422

Email karmakar@cuhk.edu.hk
Purpose: Thoracic paravertebral block (TPVB), in conjunction with intravenous sedation, is reported to provide surgical anesthesia for primary breast cancer surgery (PBCS). Although ultrasound-guided (USG) TPVB has been described, there are no reports of USG multilevel TPVB for surgical anesthesia during PBCS. The aim of this prospective observational study was to determine the feasibility of performing USG multilevel TPVB, at the T1-T6 vertebral levels (6m-TPVB), and to evaluate its efficacy in providing surgical anesthesia for PBCS.

Patients and Methods: Twenty-five female patients undergoing PBCS received an USG 6mTPVB for surgical anesthesia. Four milliliters of ropivacaine $0.5 \%$ (with epinephrine 1:200,000) was injected at each vertebral level. Dexmedetomidine infusion $\left(0.1-0.5 \mu \mathrm{g} \cdot \mathrm{kg}^{-1} \cdot \mathrm{h}^{-1}\right)$ was used for conscious sedation. Success of the block, for surgical anesthesia, was defined as being able to complete the PBCS without having to resort to rescue analgesia or convert to GA.

Results: The USG 6m-TPVB was successfully performed on all 25 patients but it was effective as the sole anesthetic in only $20 \%(5 / 25)$ of patients. The remaining $80 \%(20 / 25)$ reported pain during separation of the breast from the pectoralis major muscle and its fascia. Surgery was successfully completed using small doses of intravenous ketamine (mean total dose, $38.0 \pm 20.5 \mathrm{mg}$ ) as supplementary analgesia.

Conclusion: USG 6m-TPVB is technically feasible but does not consistently provide complete surgical anesthesia for PBCS that involves surgical dissection on the pectoralis major muscle and its fascia. Our data suggest that the pectoral nerves, which are not affected by a $6 \mathrm{~m}-\mathrm{TPVB}$, are involved with afferent nociception.

Keywords: anesthesia, analgesia, thoracic paravertebral block, mastectomy, breast, ultrasound

\section{Introduction}

Primary breast cancer surgery (PBCS), which includes simple mastectomy with sentinel lymph node biopsy (SLNB) and modified radical mastectomy (MRM) are surgical procedures that are traditionally performed under general anesthesia (GA). Thoracic paravertebral block (TPVB), in conjunction with intravenous sedation, has been used as an alternative to GA for surgical anesthesia during $\operatorname{PBCS}^{1,4}$ with improved outcomes, ${ }^{1,2,4}$ minimal complications ${ }^{1,5}$ and a high degree of patient satisfaction. ${ }^{4}$ When used for surgical anesthesia during PBCS, TPVB is performed as a multilevel injection technique (C7-T6, ${ }^{1,2} \mathrm{C} 7-\mathrm{T} 7,{ }^{4} \mathrm{~T} 1-\mathrm{T} 6,{ }^{3,6}$ and T1-T5, ${ }^{7} \mathrm{~T} 3-$ $\mathrm{T}^{5}$ ) because dermatomal anesthesia after a single-injection is variable ${ }^{8,9}$ and a multilevel injection technique produces more reliable sensory blockade than 
a single-injection. ${ }^{10} \mathrm{~A}$ review of the literature indicates that the majority of published data on TPVB for surgical anesthesia during PBCS are from a time when anatomical landmark-based techniques were used to perform multilevel TPVB. ${ }^{1,2,4,6}$ Today ultrasound guidance is frequently used for regional anesthesia including TPVB. ${ }^{11,12}$ Although several ultrasound-guided (USG) TPVB techniques have been described in the literature, ${ }^{11}$ there is a paucity of data on USG multilevel $\mathrm{TPVB}^{13}$ and, to the best of our knowledge there are no data describing USG multilevel TPVB for surgical anesthesia during PBCS. The primary objective of this study, defined a priori, was to determine the feasibility of performing USG multilevel TPVB at the T1 to T6 vertebral levels, as six separate injections (6m-TPVB), and the secondary objective was to evaluate its efficacy in providing surgical anesthesia for PBCS.

\section{Materials and Methods}

This report adheres to the applicable STROBE guidelines for reporting observational cohort studies. The study was also conducted in accordance with the Declaration of Helsinki. After clinical research ethics committee (The Joint Chinese University of Hong Kong - New Territories East Cluster Clinical Research Ethics Committee) approval and written informed consent, 25 female patients, 30-80 years old, American Society of Anesthesiologists (ASA) physical status I-III, scheduled for elective PBCS under a 6m-TPVB at the North District Hospital in Sheung Shui, Hong Kong were enrolled from November 1, 2013 to July 31, 2014) for this prospective, non-randomized, observational study. Patients with the following conditions were excluded: patient refusal, ASA physical status $>$ III, BMI $\geq 35 \mathrm{~kg} /$ $\mathrm{m}^{2}$, patients undergoing lumpectomy or partial (segmental or quadrantectomy) mastectomy, pregnancy, spinal deformity, neuromuscular disease, previous ipsilateral thoracic spine surgery, bleeding tendency or evidence of coagulopathy, history of allergy to local anesthetic drugs, skin infection at the site of needle insertion, or contraindication to regional anesthesia.

\section{Preoperative Preparation}

All patients were seen on the day before surgery and they were all fasted preoperatively. During the preoperative visit patients were instructed on the use of a numeric rating scale (NRS, $0-100 \mathrm{~mm}$ ) for pain assessment $(0=$ no pain, $100=$ worst imaginable pain). On the day of surgery, patients were admitted to the anesthetic procedure room approximately 1 hour before surgery. On arrival, intravenous access was established (18-20 G intravenous catheter) on the contralateral hand or forearm and standard monitoring (EKG, non-invasive blood pressure and $\mathrm{SaO}_{2}$ ) was instituted. "Time out" (regional anesthesia preprocedural checklist) procedure was also performed. Patients were then positioned in the lateral decubitus position with the side to be blocked uppermost, the neck slightly flexed and the upper arm resting on the pillow (Figure 1A). Oxygen $4 \mathrm{~L} / \mathrm{min}$ was administered via a facemask and expired $\mathrm{CO}_{2}$ was continuously monitored through the facemask to detect any apnea. Midazolam (1-2 mg) and ketamine (10-20 mg) were then administered intravenously for sedation and analgesia (comfort) before block placement and an intravenous infusion of dexmedetomidine $\left(0.1-0.5 \mu \mathrm{g} . \mathrm{kg}^{-1} \cdot \mathrm{h}^{-1}\right)$ was commenced (without a loading dose) and continued throughout surgery to maintain conscious sedation, whereby the patient was asleep when left undisturbed but rousable on verbal commands.

\section{The Ultrasound Scan Sequence}

The thoracic intervertebral levels (T1-T6) for the $6 \mathrm{~m}$ TPVB were identified and marked on the patients back (Figure 1A). Another line was drawn approximately 3-4 cm lateral and parallel to the midline, on the side of the block, to indicate the lateral limit for needle insertion (Figure 1A). A Philips ultrasound system (HD11XE or CX50, Philips Healthcare, Andover, MA, USA) with a low-frequency curved array transducer (C5-2, 5-2 MHz with the HD11XE or C5-1, 5-1 MHz with the CX50) was used for the ultrasound scan. Ultrasound gel was applied to the skin for acoustic coupling and the transducer was placed $2-3 \mathrm{~cm}$ lateral to the spinous process, at the target vertebral level, in the transverse plane with its orientation marker directed laterally (outward). A transverse scan of the paravertebral region was then performed in three sequential steps, over three contiguous sites over the paravertebral region (Figure 1B), for consistency and to better define the sonoanatomy relevant for the $6 \mathrm{~m}$-TPVB. The ultrasound scan sequence at each spinal level included the following steps: Step 1 (Figure 2A, position 1): the transducer was placed over the rib and costotransverse articulation. The hyperechoic outlines of the spinous process, lamina, transverse process and rib, with their acoustic shadow, were clearly delineated when the osseous anatomy was followed from a medial to lateral direction 


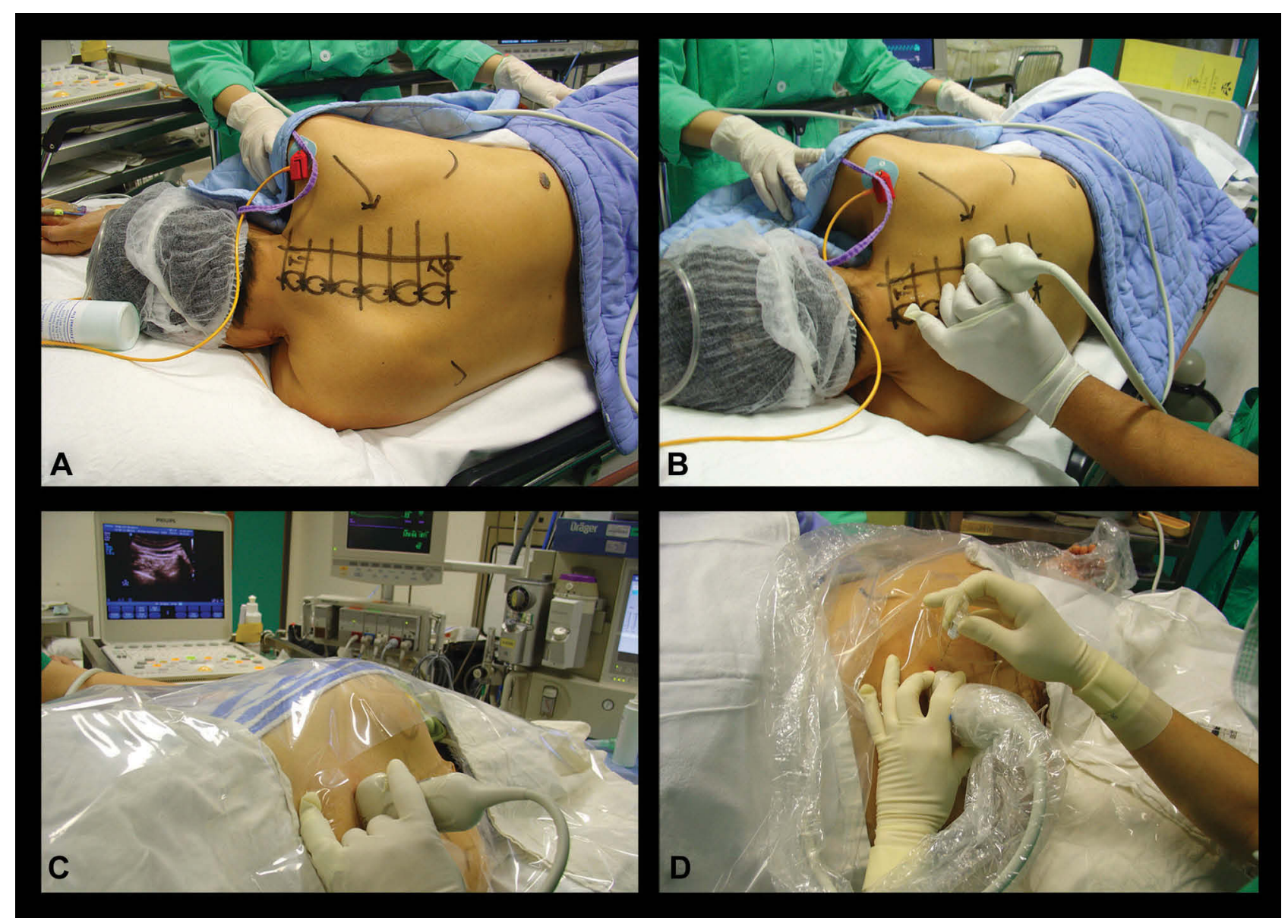

Figure I Ultrasound-guided multilevel thoracic paravertebral block for primary breast cancer surgery. Figure showing (A) position of the patient and skin markings on the back for the block, (B) position and orientation of the ultrasound transducer, (C) ergonomics used during the procedure, and (D) in-plane needle insertion from a lateral to medial direction.

(Figure 3A). Due to the large acoustic shadow produced by the spinous process, lamina, transverse process and rib complex (Figure 3A), ultrasound visualization of the paravertebral anatomy is not possible in this ultrasound view. Step 2 (Figure 2A, position 2): maintaining the same transverse orientation the transducer was slowly moved caudally until the acoustic shadow of the rib disappeared and the echogenic outlines of the lamina and transverse process with their acoustic shadow were visualized (Figure 3B). In the transverse sonogram, lateral to the transverse process, the echogenic parietal pleura and lung were visualized anteriorly, the thick echogenic superior costotransverse ligament posteriorly, and the hypoechoeic apex of the paravertebral space was interposed between the two (Figure 3B). ${ }^{12}$ Step 3 (Figure 2A, position 3 and Figure 2B): finally from the above position the transducer was tilted slightly caudally until the acoustic shadow of the transverse process disappeared and the hyperechoeic inferior articular process (IAP) with its acoustic shadow was visualized medially (Figure 3C). As in the transverse sonogram at position 2 , the superior costotransverse ligament, parietal pleura, and apex of the paravertebral space were clearly delineated but since the acoustic shadow of the transverse process was no longer visualized the superior costotransverse ligament and outlines of the paravertebral space were better defined (Figure 3C). The latter ultrasound window represents the paramedian transverse view of the thoracic paravertebral anatomy, as imaged through the inter-transverse space (Figure 4), which was used as the target ultrasound window for the paravertebral injections in this study.

\section{Ultrasound-Guided Multilevel Thoracic Paravertebral Block}

The $6 \mathrm{~m}$-TPVB was performed using real-time ultrasound guidance and by starting at the T6 vertebral level (Figure 5) after which each subsequent paravertebral injection was 


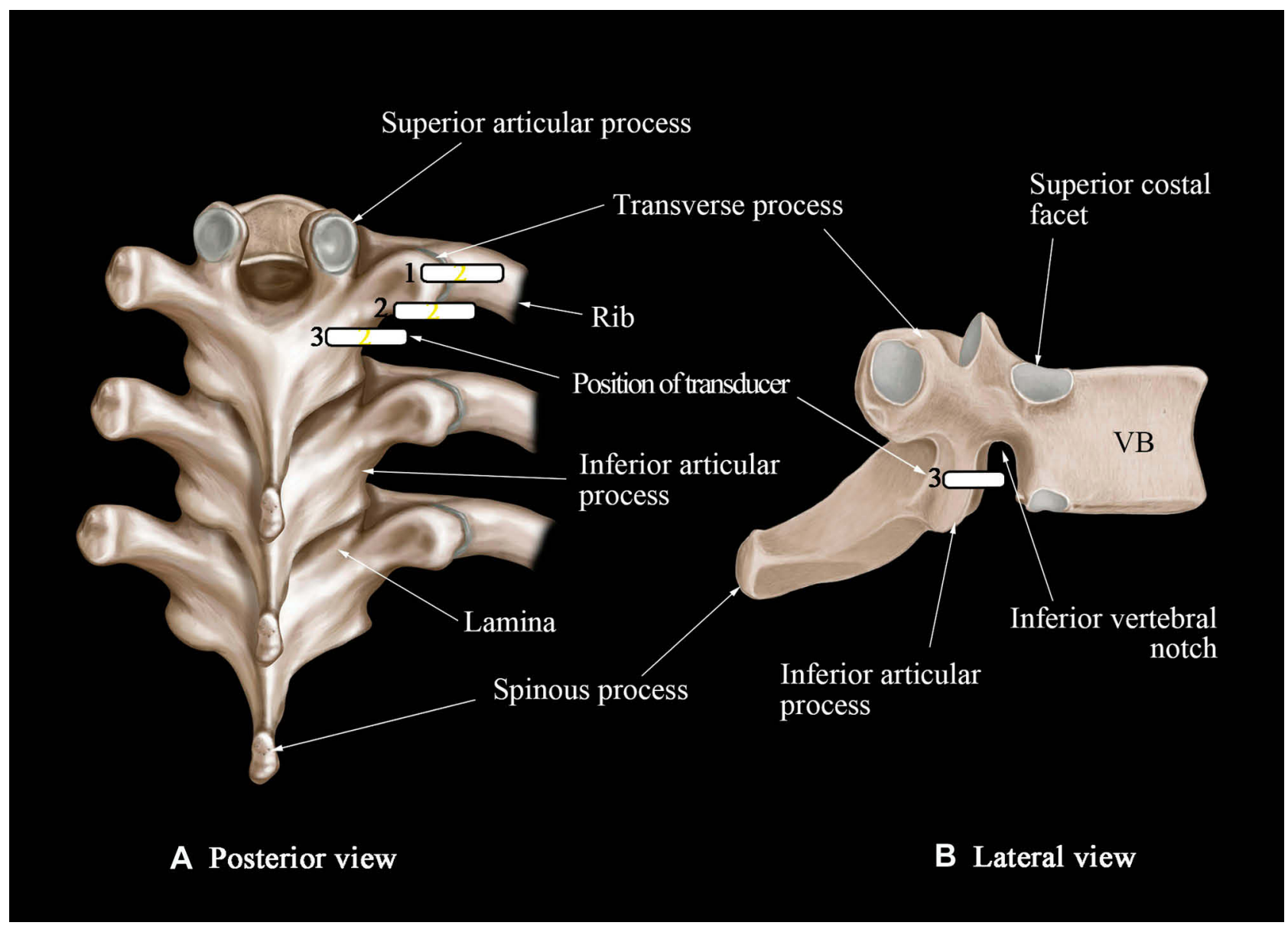

Figure 2 (A) Figure illustrating the various positions of the ultrasound transducer, relative to the osseous structures of the thoracic spine, during the ultrasound scan sequence described in this report. Position I: at the level of the rib and transverse process; position 2: at the level of the transverse process; position 3: at the level of the inferior articular process. (B) Note the relationship of the inferior articular process to the inferior vertebral notch and the intervertebral foramen.

performed in a caudo-cephalad direction. All paravertebral injections during the study were performed or supervised by a single experienced operator (MKK) with more than 25 years of experience performing TPVB. The target transverse ultrasound window (described above) was acquired and optimized at each thoracic level (Figures 5-6) before the paravertebral injection. Thereafter under strict aseptic precautions (Figure 1C) and under local infiltration (Lignocaine $1 \%, 1 \mathrm{~mL}$ at each level) a SonoTAP nerve block needle (Pajunk, 22G, $80 \mathrm{~mm}$, Pajunk, Geisingen, Germany) was inserted in-plane and slowly advanced from a lateral to medial direction (Figure 1D, Figures 5-6) until its tip was located at the apex of the paravertebral space. Sonographic criteria were used to confirm correct needle tip position: 1) visualizing the needle tip at the apex of the paravertebral space (Figure 5B); and/or 2) anterior displacement of the parietal pleura after a test bolus injection of $1-2 \mathrm{~mL}$ of normal saline. ${ }^{12}$ Four $\mathrm{mL}$ of ropivacaine $0.5 \%$ (with 1:200,000 adrenaline) was then slowly injected through the nerve block needle while anterior displacement of the parietal pleura (Figure 5C) was closely monitored. The procedure was repeated at T5-T1 (Figures 5-6) with the same volume of ropivacaine $0.5 \%$ (with 1:200,000 epinephrine) but the total volume was limited to $25 \mathrm{~mL}(125 \mathrm{mg}$ ) for the entire procedure. Since visualization of the parietal pleura at the $\mathrm{T} 1$ level is difficult ${ }^{14}$ the ultrasound scan technique was modified by tilting the transducer slightly caudad to visualize the parietal pleura (Figure 6G). Thereafter the procedure was identical to that at the other thoracic levels except that the block needle had to be inserted with a slightly steeper angle and in an oblique direction at the T1 level (Figure 6H). No formal sensory assessment was performed since patients were sedated. Surgery started about 25-30 minutes after the last paravertebral injection. The $6 \mathrm{~m}$-TPVB technique was considered a success if anterior displacement of the pleura was visualized after the local anesthetic (LA) injection at all six levels and patients did not complain of pain during the surgical incision. 


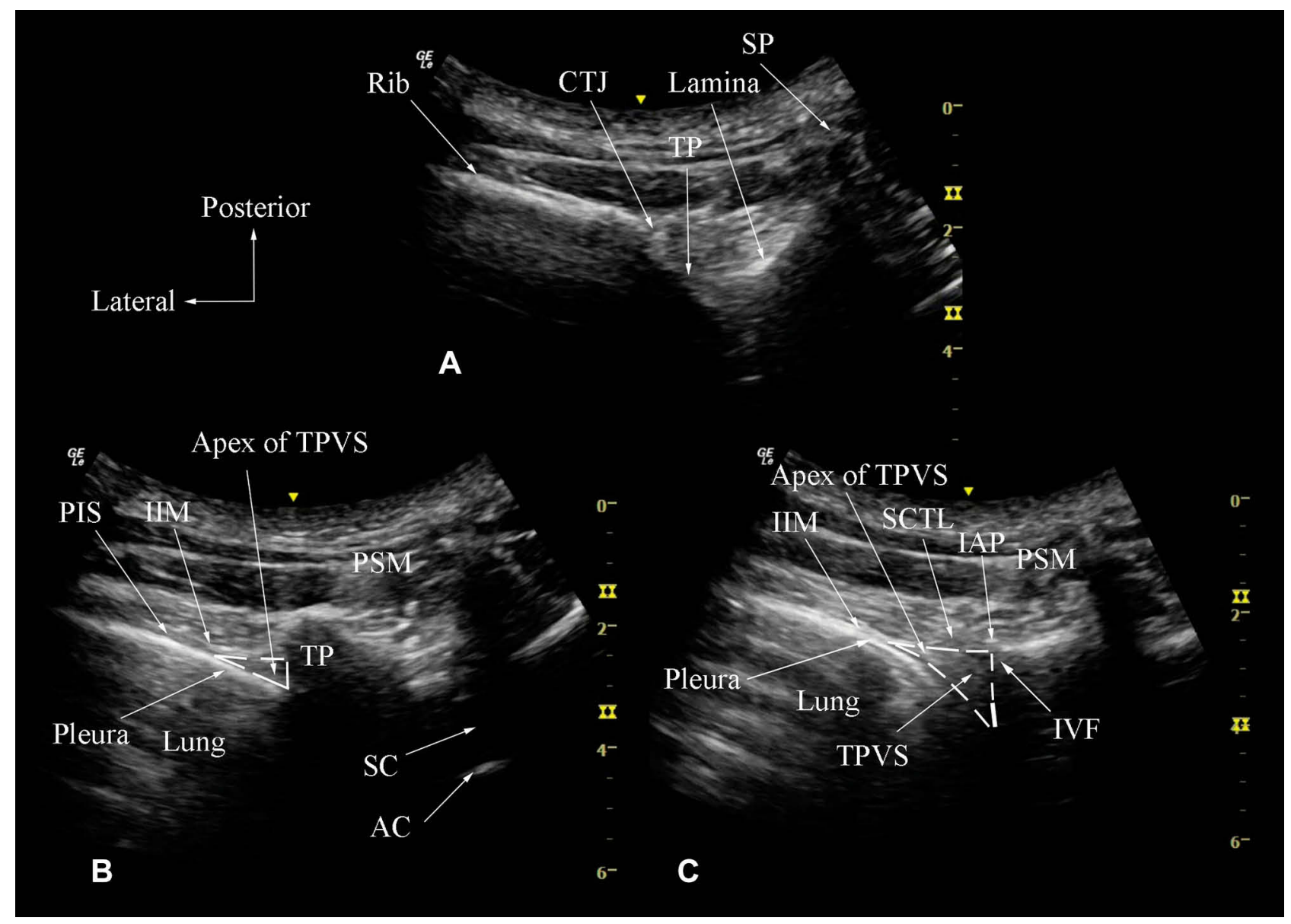

Figure 3 Paramedian transverse sonograms of the thoracic paravertebral region with the ultrasound beam being insonated (A) at the level of the rib and transverse process (position I in Figure 2); (B) at the level of the transverse process (position 2 in Figure 2), and (C) at the level of the inferior articular process (position 3 in Figure 2). Abbreviation: SP, spinous process; TP, transverse process; CTJ, costotransverse junction; PSM, paraspinal muscles; TPVS, thoracic paravertebral space; IIM, internal intercostal membrane; PIS, posterior intercostal space; SC, spinal canal; AC, anterior complex; IAP, inferior articular process; SCTL, superior costotransverse ligament; IVF, intervertebral foramen.

\section{Intraoperative Management}

Midazolam (1 mg, i.v. bolus as needed) and dexmedetomidine infusion $\left(0.1-0.5 \mu \mathrm{g} \cdot \mathrm{kg}^{-1} \cdot \mathrm{h}^{-1}\right)$ was used to maintain conscious sedation during surgery. Oxygen $(4 \mathrm{~L}$. $\mathrm{min}^{-1}$ ) and apnea monitoring (expired $\mathrm{CO}_{2}$ ) was continued via the facemask or nasal cannula throughout surgery. Patients were also offered a headphone to listen to music from a portable music device. Surgery usually commenced 25-30 mins after the last paravertebral injection. The PBCS was performed via a single transverse elliptical skin incision, extending from the medial aspect of the breast (close to the sternum) to the anterior margin of the latissimus dorsi muscle, and included the nipple-areolar complex. Mastectomy skin flaps were raised using electrocautery and the breast parenchyma with the fascia overlying the pectoralis major muscle were removed from the chest wall. The sentinel lymph node biopsy and axillary lymph node dissection were also performed through the same skin incision. Before the surgical incision the surgeon ensured that the patient did not respond to painful stimulus, by pinching the skin with a tooth forceps, at the axilla (intercostobrachial nerve, T2), along the skin markings for the surgical incision (T3-T5) and along the inframammary crease (T6). If patients reported pain to the painful stimulus then the surgery was withheld for 5-10 minutes, to allow for any further progression of the sensory blockade, and the test was repeated by the surgeon. If the patient still reported pain then the block was considered a failure and GA was administered with induction of unconsciousness, intravenous opioids and airway support. If patients did not report pain to the painful stimulus then surgery commenced and ketamine (10-20 mg i.v. bolus) was administered as rescue analgesia for any pain reported thereafter, up to an arbitrary maximum of $100 \mathrm{mg}$ (for the 


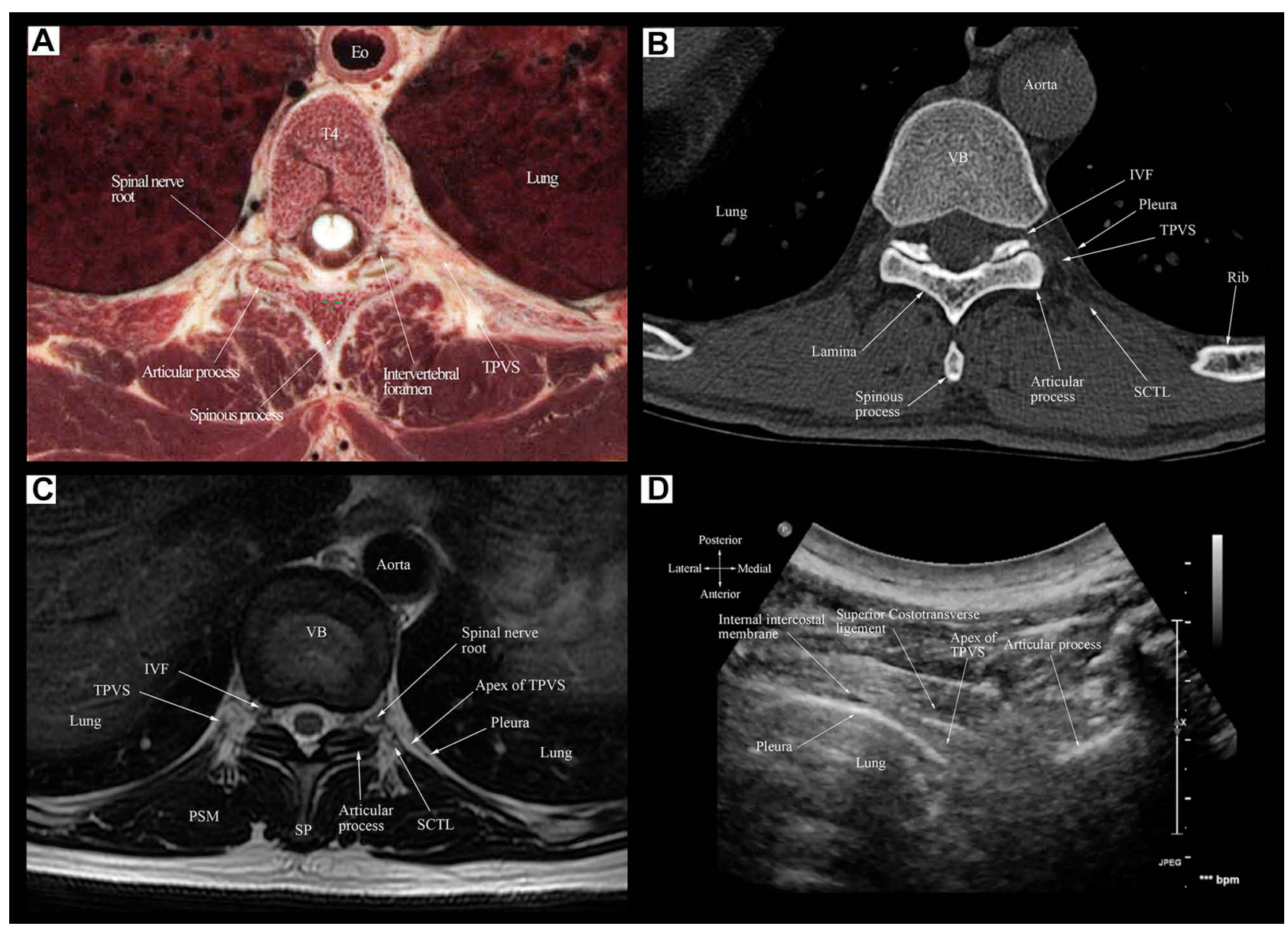

Figure 4 Correlative transverse cadaver (A), CT (B), MRI (C), and ultrasound (D) images of the thoracic paravertebral region from the level of the vertebral body and inferior articular process corresponding to the level at which the paramedian transverse scan was performed (Figure 2, position 3).

Abbreviations: Eo, oesophagus; TPVS, thoracic paravertebral space; VB, vertebral body; IVF, intervertebral foramen; SCTL, superior costotransverse ligament; SP, spinous process.

study), after which the block was considered a failure and converted to GA. Any drop in blood pressure $>20 \%$ compared to the baseline value was defined as hypotension and treated with phenylephrine (100 $\mu \mathrm{g}$ i.v. bolus). Dexmedetomidine infusion was discontinued 15-20 minutes before the end of surgery.

\section{Management in the PACU}

After surgery, patients were transferred to the post anesthetic care unit (PACU) for postoperative monitoring before they were discharged to the surgical ward. Postoperative pain was assessed using a NRS described above. If NRS pain score was greater than 40 at rest or patient requested additional analgesia, morphine $1 \mathrm{mg}$ was administered i.v. and repeated every 5 minutes, as deemed necessary, until NRS pain score was $\leq 30$. If patient reported nausea that persisted for more than 10 minutes or vomited more than twice, ondansetron $4 \mathrm{mg}$ was administered i.v. to treat the postoperative nausea and vomiting. Aldrete post-anesthetic recovery score ${ }^{15}$ was also recorded at two time points: 1) on arrival to the PACU; and 2) at discharge from the PACU.

\section{Outcome Measures}

An independent observer (research nurse) recorded all outcomes variables. The primary aim of this study was to determine the feasibility of performing the USG 6m-TPVB. The secondary aim was to evaluate the efficacy of the $6 \mathrm{~m}$ TPVB, in providing surgical anesthesia for PBCS and defined as being able to complete the scheduled PBCS without having to resort to rescue analgesia or convert to GA. Other secondary outcomes recorded included any complication directly related to the technique or local anesthetic injection (vascular puncture, pleural puncture, pneumothorax or local anesthetic toxicity). Intraoperative requirements of ketamine, dexmedetomidine, and phenylephrine were also quantified from the data recorded. 


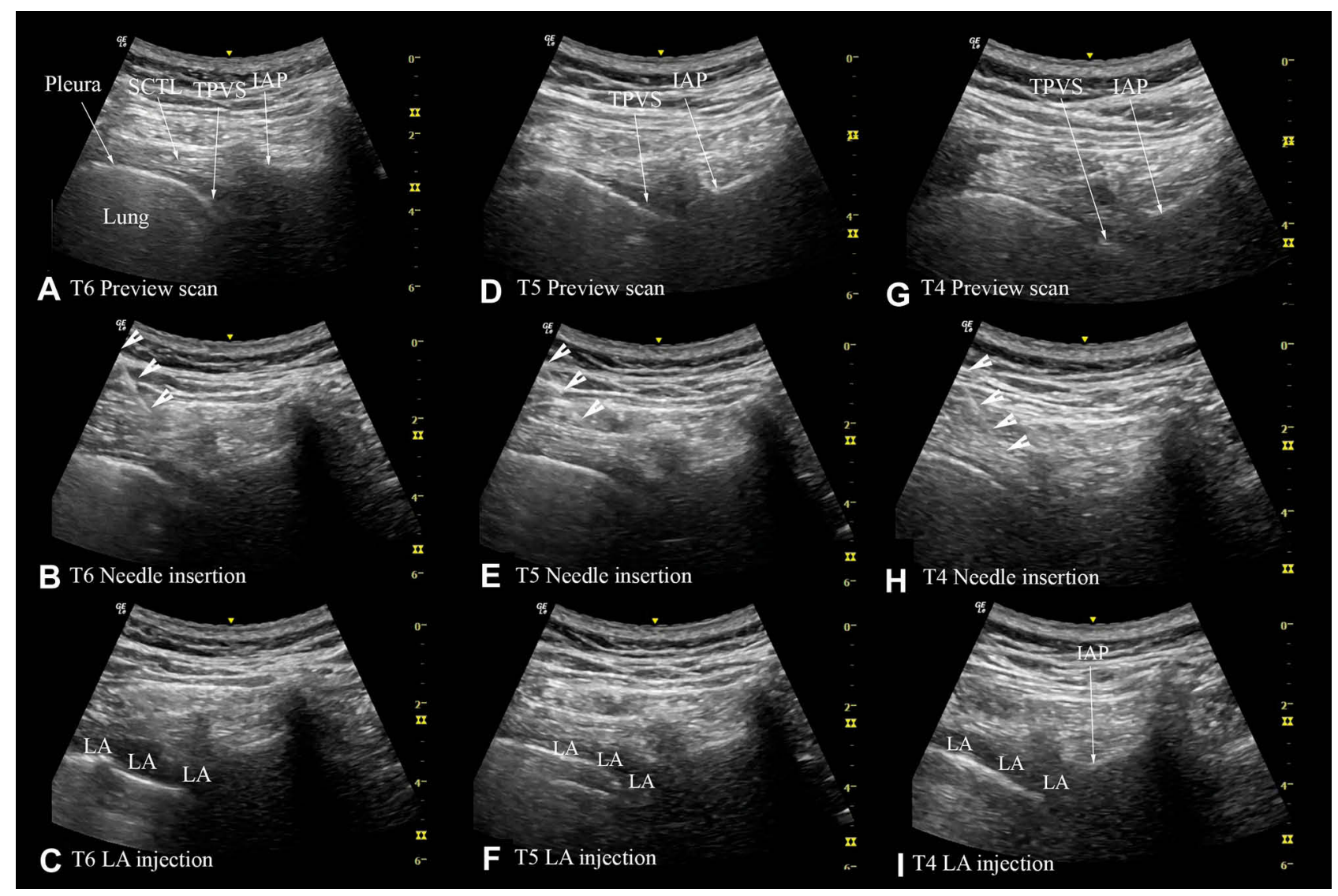

Figure $5 \mathrm{~A}$ sequence of transverse sonograms demonstrating ultrasound guided 6m-TPVB at the T6-T4 vertebral levels using the transverse in-plane technique at the level of the inferior articular process in the same patient. At each vertebral level, T6 (A-C), T5 (D-F) and T4 (G-I). (A) represents the preview scan, (B) the sonogram during the in-plane needle insertion; and (C) the sonogram after the local anaesthetic (LA) injection. The needle is represented using white arrow heads. Note the widening of the paravertebral space and anterior displacement of the pleura after the LA injection at all three levels.

Abbreviations: IAP, inferior articular process; TPVS, thoracic paravertebral space; SCTL, superior costotransverse ligament.

\section{Statistics}

Twenty-five patients were chosen for this study because we considered it to be an adequate cohort to evaluate the defined outcomes of this study. Data were analyzed using the Statistical Package for the Social Sciences (SPSS for Windows, Version 20, IBM Corporation, Armonk, NY, USA). Data are presented descriptively as a mean (SD) when normally distributed and as a median [interquartile range, IQR] when not normally distributed. Categorical variables are presented as a frequency and percentage (n, \%).

\section{Results}

The USG 6m-TPVB was successfully performed on all 25 patients (Table 1) using the technique described but the paravertebral injection at the $\mathrm{T} 1$ level was technically more challenging than that at the T2 to T6 levels. Clinical parameters relating to the $6 \mathrm{~m}$-TPVB are presented in Table 2 . Surgery lasted for an average of $99.7 \pm 28.7$ minutes and the $6 \mathrm{~m}$-TPVB was effective as the sole anesthetic for the PBCS in only $20 \%(5 / 25)$ of patients. In the remaining $80 \%(20 / 25)$ of patients it provided incomplete anesthesia with patients reporting pain or discomfort during surgical separation of the breast from the fascia of the pectoralis major muscle and/ or washout of the surgical wound with warm normal saline towards the end of surgery. Surgery was successfully completed using small bolus doses of intravenous ketamine (mean total dose, $38.0 \pm 20.5 \mathrm{mg}$ ) as supplementary analgesia (Table 1). Patients reported no pain at rest on arrival (median NRS, $0[0-0]$ ), or discharge from the PACU (median NRS, 0 [0-1]). Hypotension, requiring i.v. phenylephrine, developed in $8 / 25$ (32\%) patients. Otherwise, there were no complications directly related to the technique of $6 \mathrm{~m}$-TPVB or the local anesthetic injection, and recovery from the paravertebral block was uneventful.

\section{Discussion}

This study aimed to determine the feasibility of performing USG 6m-TPVB, and to evaluate its efficacy in 


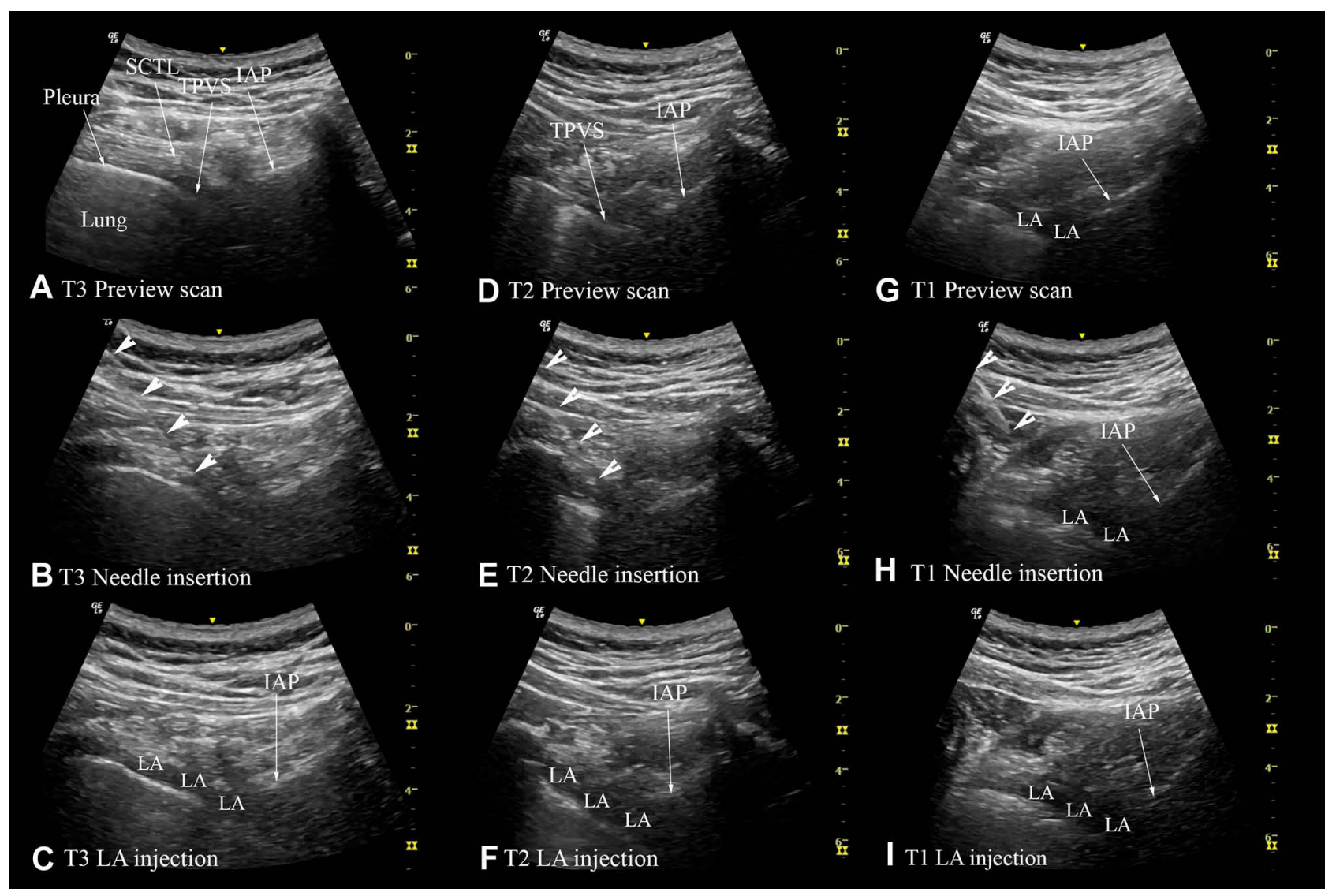

Figure 6 A sequence of transverse sonograms demonstrating ultrasound-guided 6m-TPVB at the T3-TI vertebral levels (same patient as in Figure 5) using the transverse inplane technique at the level of the inferior articular process. At each vertebral level, T3 (A-C), T2 (D-F) and TI (G-I). (A) represents the preview scan, (B) represents the sonogram during the in-plane needle insertion; and (C) represents the sonogram after the local anaesthetic (LA) injection. The needle is represented using arrow heads. Note the widening of the thoracic paravertebral space (TPVS) in the preview scan at the TI level (G) consequent to the spread of LA from the contiguous space (T2). There is also widening of the paravertebral space and anterior displacement of the pleura after the LA injection at all three levels.

Abbreviations: IAP, inferior articular process; SCTL, superior costotransverse ligament.

providing surgical anesthesia for PBCS. USG 6m-TPVB was technically feasible but did not consistently provide complete surgical anesthesia for PBCS. We believe this is the first report to describe the use of an USG 6m-TPVB, in conjunction with conscious sedation, for surgical anesthesia during PBCS.

Table I Demographic Data

\begin{tabular}{|l|l|}
\hline \multicolumn{2}{|l|}{ Demographic Data (n=25) } \\
\hline I. Age (years) & $59.0 \pm 13.0$ \\
2. ASA (I/II/III) & $10 / 1 \mathrm{I} / 4$ \\
3. Height (cm) & $158.0 \pm 3.8$ \\
4. Weight (kg) & $58.2 \pm 11.8$ \\
5. BMI (kg.m²) & $23.3 \pm 4.8$ \\
\hline
\end{tabular}

Notes: Data are presented as mean \pm SD for age, height, weight and BMI. ASA status is presented as a frequency ( $n$ ).

Abbreviations: ASA, American Society of Anesthesiologists; BMI, body mass index.
A transverse ultrasound scan through the thoracic intertransverse space and at the level of the IAP was used to perform the paravertebral injections. The block needle was inserted in-plane and from a lateral to medial direction. Other researchers have also described USG TPVB, albeit in cadavers, ${ }^{16}$ using a transverse scan at the level of the IAP. ${ }^{11,16}$ However, the difference between our technique and that described by others ${ }^{11,16}$ is that while others deliberately contact the IAP and then maneuver the needle laterally into the paravertebral space close to the intervertebral foramen (IVF), ${ }^{11,16}$ we directly place the needle tip at the apex of the paravertebral space. This is done to avoid placing the needle close to the IVF, since a "medial injection" $" 17$ may predispose to neuraxial complications. ${ }^{18,19}$ Also since the block needle is advanced tangential to the parietal pleura ${ }^{12}$ unintentional pleural puncture is unlikely. ${ }^{12}$ Currently there are no published data on the safety and efficacy of an USG TPVB using a transverse scan at the level of the IAP. ${ }^{11,16}$ Our 
Table 2 Clinical Parameters and Outcomes

\begin{tabular}{|l|l|}
\hline \multicolumn{2}{|l|}{ Parameters (n=25) } \\
\hline I. Type of breast surgery* (a/b/c) & $3 / 6 / 16$ \\
2. Side of surgery (right/left) & $12 / 13$ \\
3. Dose of midazolam before block placement $(\mathrm{mg})$ & $1.7 \pm 0.8$ \\
4. Dose of ketamine before block placement $(\mathrm{mg})$ & $16.9 \pm 7.5$ \\
5. Total duration of surgery (minutes) & $99.7 \pm 28.7$ \\
6. Minimum dexmedetomidine infusion rate $(\mu \mathrm{g} / \mathrm{kg} / \mathrm{h})$ & $0.2[0.1-0.3]$ \\
7. Maximum dexmedetomidine infusion rate $(\mu \mathrm{gg} / \mathrm{kg} / \mathrm{h})$ & $0.33[0.3-0.5]$ \\
8. Supplementary midazolam during surgery $(\mathrm{yes} / \mathrm{no})$ & $12 / 25(48 \%)$ \\
9. Dose of midazolam used during surgery $(\mathrm{mg})$ & $2.0(0.9)$ \\
I0. Ketamine for rescue analgesia (yes/no) & $20 / 25(80 \%)$ \\
II. Dose of ketamine used as rescue analgesia $(\mathrm{mg})$ & $38.0 \pm 20.5$ \\
I2. Phenylephrine for hypotension (yes/no) & $8 / 25(32 \%)$ \\
I3. Aldrete score on arrival to PACU & $8.9 \pm 1.3$ \\
I4. Aldrete score at discharge from PACU & $9.7 \pm 0.5$ \\
I5. NRS pain score on arrival to PACU & $0[0-0]$ \\
I6. NRS pain score at discharge from PACU & $0[0-1]$ \\
\hline
\end{tabular}

Notes: Continuous data are presented as mean \pm SD, except for the infusion rate of dexmedetomidine and NRS (numeric rating score) pain score, which are presented as median [IQR]. Categorical data are described as a frequency, n (\%). *Type of surgery: a; Simple mastectomy with sentinel lymph node biopsy; b; simple mastectomy with sentinel lymph node biopsy and axillary lymph node dissection; c; modified radical mastectomy.

Abbreviation: PACU, post-anaesthetic care unit.

preliminary experience is encouraging but future research should establish its safety and efficacy in a larger patient population.

The USG 6m-TPVB was effective as the sole anesthetic for the PBCS in only $20 \%$ of patients. In the remaining $80 \%$ it provided incomplete anesthesia with patients reporting pain or discomfort, 1) during separation of the breast (base) from the fascia of the pectoralis major muscle; and/or 2) washout of the surgical wound with warm normal saline. There are no comparable data but our results contradict previous reports of multilevel $\mathrm{TPVB}^{1,7}$ being successfully used, in conjunction with intravenous sedation, as the sole anesthetic technique for PBCS. ${ }^{1,7}$ The reason for this discrepancy is not clear but the success rate of a multilevel TPVB for surgical anesthesia during PBCS varies from $80-100 \%{ }^{1,3,5,20,21}$ Some form of supplementation (LA infiltration, systemic opioids or ketamine) is required to complete surgery in $10-27 \%^{1,5}$ and conversion to GA is required in $3-16 \%{ }^{1,3,5,21}$ Reasons for failure or need for supplementary anesthesia or analgesia are poorly documented and often attributed to technical difficulties, ${ }^{1,3}$ incomplete paravertebral block, ${ }^{2,3}$ or patient anxiety and restlessness. ${ }^{3}$ None of the previous reports ${ }^{1,5}$ describe the pectoralis major muscle or its fascia as a source of pain during surgery. All paravertebral injections in this study were deemed to have been successful since it was ultrasound guided and sonographic evidence of pleural displacement ${ }^{12}$ and spread of the LA within the paravertebral space, was confirmed at every vertebral level (T1T6). Moreover, none of our patients reported pain during the skin incision or axillary dissection. Therefore, we believe the cause of pain during surgery was not due to a failure of the $6 \mathrm{~m}$-TPVB per se but due to afferent nociception from an area of the breast, ie, the pectoralis major muscle and its fascia, that is innervated by the medial and lateral pectoral nerves and not affected by a 6m-TPVB. Our results support Woodworth et al's ${ }^{22}$ assertion that breast surgery involving the pectoral muscles, eg, MRM, requires more than just a $6 \mathrm{~m}$-TPVB for surgical anesthesia or analgesia. ${ }^{22}$ Given the above, why the 6m-TPVB was still effective as the sole anesthetic in $20 \%$ of our patients is not clear, and probably multifactorial, but extensive epidural spread in these cases cannot be excluded.

The majority of patients experienced pain during separation of the breast (base) from the pectoralis major muscle and its fascia. This indicates that the pectoral nerves are involved with afferent nociception during PBCS. This is at odds with current understanding because the pectoral nerves are often described as pure motor nerves. ${ }^{22,23}$ This concept is questionable since the pectoral nerves carry nociceptive, ${ }^{22,24}$ proprioceptive, ${ }^{22,24}$ and postganglionic sympathetic ${ }^{22}$ nerve fibers. Currently there is a paucity of data on the effectiveness of a pectoral nerve block for anesthesia or analgesia during PBCS. ${ }^{25}$ Pawa et $\mathrm{al}^{25}$ describe a combined single-injection TPVB and PECS-2 block technique for breast surgery under sedation. However, it is noteworthy that the majority of patients in Pawa et al's ${ }^{25}$ series underwent relatively minor breast surgery $^{25}$ and a significant number of patients (11/16, $69 \%$ ) still required supplementary fentanyl, ${ }^{25}$ one required LA supplementation, and one conversion to GA. ${ }^{25}$ Therefore it is questionable if the combined singleinjection TPVB and PECS-2 block technique ${ }^{25}$ will consistently provide surgical anesthesia for PBCS and future research to confirm this is warranted. Also future research should evaluate the role of a pectoral nerve block in improving surgical anesthesia produced by a $6 \mathrm{~m}$-TPVB for PBCS that involves pectoral dissection.

There were no major complications directly related to the USG 6m-TPVB or LA injection. Hypotension developed in $32 \%$ of patients in this study, which is higher than that previously reported $(4 \%) .{ }^{26}$ We believe this is because patients in our series were sedated, but extensive epidural spread of the LA cannot be ruled out. 


\section{Limitations}

There are limitations in our study. It was non-randomized, lacked a comparator group and patients were of low BMI. We did not randomize our patients because this was the first study to evaluate the feasibility and effectiveness of an USG $6 \mathrm{~m}$-TPVB for surgical anesthesia during PBCS. The BMI of patients studied was low but is consistent with the typical patient at the investigator's institution. Therefore, our results may not apply to the obese. We also did not perform any formal sensory assessment after the 6m-TPVB because patients were sedated and had received ketamine for analgesia during block placement. Instead, surrogate sonographic and clinical signs (described above) were used to confirm successful paravertebral injection and adequacy of the block at the T1-T6 levels.

\section{Conclusions}

We have demonstrated that USG $6 \mathrm{~m}-\mathrm{TPVB}$ is technically feasible but does not consistently provide complete surgical anesthesia for PBCS that involves surgical dissection on the pectoralis major muscle and its fascia. The need for supplementary analgesia during pectoral dissection indicates that the pectoral nerves, which are not affected by a 6m-TPVB, are involved with afferent nociception. Future research to evaluate if a pectoral nerve block improves the surgical anesthesia produced by a $6 \mathrm{~m}-$ TPVB is warranted.

\section{Data Sharing Statement}

Data pertaining to this study will be available for sharing for a period of 3 years after the date of publication of this manuscript by writing to the corresponding author. The request for the data must be for research purpose and on submission of the following documents: a cover letter, study protocol, research ethics approval and clinical trial registration details.

\section{IRB Contact Information}

Joint Chinese University of Hong Kong-New Territories East Cluster Clinical Research Ethics Committee, CREC Reference No: CRE-2013.380, Ethics approval date: October 3, 2013. Contact CRE Officer Tel: +852 3505 3935 .

\section{Acknowledgments}

We would like to thank the operating room and PACU staff at the North District Hospital, Sheung Shui, NT,
Hong Kong for their assistance during the conduct of this study. The anatomic image in Figure 4A (free online access) is courtesy of the Visible Human Server at Ecole Polytechnique Fédérale de Lausanne, Visible Human Visualisation Software, and Gold Standard Multimedia. The CT (Figure 4B) and MRI (Figure 4C) images were rendered from datasets in the authors' archive.

\section{Funding}

This research work was locally funded by the Department of Anaesthesia and Intensive Care, The Chinese University of Hong Kong, Prince of Wales Hospital, Shatin, New Territories, Hong Kong, SAR.

\section{Disclosure}

The authors report no conflicts of interest in this work.

\section{References}

1. Coveney E, Weltz CR, Greengrass R, et al. Use of paravertebral block anesthesia in the surgical management of breast cancer experience in 156 cases. Ann Surg. 1998;227:496-501. doi:10.1097/ 00000658-199804000-00008

2. Greengrass R, O’Brien F, Lyerly K, et al. Paravertebral block for breast cancer surgery. Can J Anaesth. 1996;43(8):858-861. doi:10.10 07/BF03013039

3. Najarian MM, Johnson JM, Landercasper J, Havlik P, Lambert PJ, McCarthy D. PVB: an alternative to GA in breast cancer sugery. Am J Surg. 2003;69:213-218.

4. Weltz CR, Greengrass RA, Lyerly HK. Ambulatory surgical management of breast carcinoma using paravertebral block [see comments]. Ann Surg. 1995;222(1):19-26. doi:10.1097/00000658-19950700000004

5. Das S, Bhattacharya P, Mandal MC, Mukhopadhyay S, Basu SR, Mandol BK. Multiple-injection thoracic paravertebral block as an alternative to general anaesthesia for elective breast surgeries: a randomised controlled trial. Indian J Anaesth. 2012;56(1):27-33. doi:10.4103/0019-5049.93340

6. D'Ercole FJ, Scott D, Bell E, Klein SM, Greengrass RA. Paravertebral blockade for modified radical mastectomy in a pregnant patient. Anesth Analg. 1999;88(6):1351-1353. doi:10.121 3/00000539-199906000-00029

7. Naja MZ, Ziade MF, Lonnqvist PA. Nerve-stimulator guided paravertebral blockade vs. general anaesthesia for breast surgery: a prospective randomized trial. Eur $J$ Anaesthesiol. 2003;20 (11):897-903. doi:10.1097/00003643-200311000-00007

8. Cheema S, Richardson J, McGurgan P. Factors affecting the spread of bupivacaine in the adult TPV space. Anaesthesia. 2003;58 (7):684-711. doi:10.1046/j.1365-2044.2003.03189_1.x

9. Karmakar MK, Critchley LA, Ho AM, Gin T, Lee TW, Yim AP. Continuous thoracic paravertebral infusion of bupivacaine for pain management in patients with multiple fractured ribs. Chest. 2003;123 (2):424-431. doi:10.1378/chest.123.2.424

10. Naja ZM, El-Rajab M, Al-Tannir MA, et al. Thoracic paravertebral block: influence of the number of injections. Reg Anesth Pain Med. 2006;31(3):196-201. doi:10.1016/j.rapm.2005.12.004

11. Krediet AC, Moayeri N, van Geffen GJ, et al. Different approaches to ultrasound-guided thoracic paravertebral block: an illustrated review. Anesthesiology. 2015;123(2):459-474. doi:10.1097/ALN.000000000 0000747 
12. Shibata Y, Nishiwaki K. Ultrasound-guided intercostal approach to thoracic paravertebral block. Anesth Analg. 2009;109(3):996-997. doi:10.1213/ane.0b013e3181af7e7b

13. Abdallah FW, Morgan PJ, Cil T, et al. Ultrasound-guided multilevel paravertebral blocks and total intravenous anesthesia improve the quality of recovery after ambulatory breast tumor resection. Anesthesiology. 2014;120(3):703-713. doi:10.1097/ALN.00004361 17.52143.bc

14. Hara K, Sakura S, Nomura T, Saito Y. Ultrasound guided thoracic paravertebral block in breast surgery. Anaesthesia. 2009;64 (2):223-224. doi:10.1111/j.1365-2044.2008.05843.x

15. Aldrete JA, Kroulik D. Postanesthetic recovery score. Anesth Analg. 1970;49:924-934.

16. Luyet C, Herrmann G, Ross S, et al. Ultrasound-guided thoracic paravertebral puncture and placement of catheters in human cadavers: where do catheters go? Br J Anaesth. 2011;106(2):246-254. doi:10.1093/bja/aeq309

17. Shaw WM, Hollis NY. Medial approach for paravertebral somatic nerve block. JAMA. 1952;148(9):742-744. doi:10.1001/jama.1952. $62930090012012 \mathrm{e}$

18. Evans PJ, Lloyd JW, Wood GJ. Accidental intrathecal injection of bupivacaine and dextran. Anaesthesia. 1981;36(7):685-687. doi:10.1111/j.1365-2044.1981.tb08781.x

19. Sharrock NE. Postural headache following thoracic somatic paravertebral nerve block. Anesthesiology. 1980;52(4):360-362. doi:10.109 7/00000542-198004000-00017
20. Tahiri Y, Tran DQ, Bouteaud J, et al. General anaesthesia versus thoracic paravertebral block for breast surgery: a meta-analysis. J Plast Reconstr Aesthet Surg. 2011;64(10):1261-1269. doi:10.10 16/j.bjps.2011.03.025

21. Thavaneswaran P, Rudkin GE, Cooter RD, Moyes DG, Perera CL, Maddern GJ. Paravertebral block for anesthesia: a systematic review. Anesth Analg. 2010;110(6):1740-1744. doi:10.1213/ANE.0b013e3 $181 \mathrm{da} 82 \mathrm{c} 8$

22. Woodworth GE, Ivie RMJ, Nelson SM, Walker CM, Maniker RB. Perioperative breast analgesia: a qualitative review of anatomy and regional techniques. Reg Anesth Pain Med. 2017;42(5):609-631. doi:10.1097/AAP.0000000000000641

23. Porzionato A, Macchi V, Stecco C, Loukas M, Tubbs RS, De Caro R. Surgical anatomy of the pectoral nerves and the pectoral musculature. Clin Anat. 2012;25(5):559-575. doi:10.1002/ca.21301

24. Mense S. Nociception from skeletal muscle in relation to clinical muscle pain. Pain. 1993;54(3):241-289. doi:10.1016/0304-3959(93) 90027-M

25. Pawa A, Wight J, Onwochei DN, et al. Combined thoracic paravertebral and pectoral nerve blocks for breast surgery under sedation: a prospective observational case series. Anaesthesia. 2018;73 (4):438-443. doi:10.1111/anae.14213

26. Naja Z, Lonnqvist PA. Somatic paravertebral nerve blockade. Incidence of failed block and complications. Anaesthesia. 2001;56 (12):1184-1188. doi:10.1046/j.1365-2044.2001.02084-2.x
Journal of Pain Research

\section{Publish your work in this journal}

The Journal of Pain Research is an international, peer reviewed, open access, online journal that welcomes laboratory and clinical findings in the fields of pain research and the prevention and management of pain. Original research, reviews, symposium reports, hypothesis formation and commentaries are all considered for publication. The manuscript management system is completely online and includes a very quick and fair peer-review system, which is all easy to use. Visit http:// www.dovepress.com/testimonials.php to read real quotes from published authors. 